Gefässchirurgie 2019 • 24:388-398 https://doi.org/10.1007/s00772-019-0550-9 Online publiziert: 12. August 2019 (c) Springer Medizin Verlag GmbH, ein Teil von Springer Nature 2019

\author{
M. Storck ${ }^{1,3} \cdot$ J. Dissemond ${ }^{1,2} \cdot$ V. Gerber ${ }^{1,2} \cdot$ M. Augustin ${ }^{1,2} \cdot$ Expertenrat $^{2}$ \\ Strukturentwicklung Wundmanagement \\ 'Deutscher Wundrat GmbH, Hamburg, Deutschland \\ ${ }^{2}$ Initiative Chronische Wunden e.V., Quedlinburg, Deutschland \\ ${ }^{3}$ Klinik für Gefäß- und Thoraxchirurgie, Städt. Klinikum Karlsruhe, Karlsruhe, Deutschland
}

\section{Kompetenzlevel in der Wundbehandlung}

\section{Empfehlungen zur Verbesserung der Versorgungsstruktur für Menschen mit chronischen Wunden in Deutschland}

\section{Einleitung}

In Deutschland dauert es im Durchschnitt 3,5 Jahre, bis ein Patient mit einer chronischen Wunde fachärztlich behandelt und einer kausalen Therapie unterzogen wird $[1,2]$. Frühere nationale Expertenrunden haben sich bereits mit der Frage einer geeigneten Dokumentation von Therapie und Outcome beschäftigt [3]. Eine Wunde, die nach 8 Wochen nicht abgeheilt ist, wird per Definition als „chronisch“ bezeichnet. Unabhängig von dieser zeitlich orientierten Definition, gibt es Wunden, die von Beginn an als chronisch anzusehen sind, da ihre Behandlung eine Therapie der weiterhin bestehenden Ursache erfordert. Hierzu gehören beispielsweise das diabetische Fußulkus, Wunden bei PAVK, Ulcus cruris venosum oder Dekubitus.

Fest steht, dass die Chance, eine Abheilung zu erreichen, umso höher ist, je früher eine chronische Wunde diagnostiziert und die fachgerechte und medizinisch notwendige Therapie eingeleitet wird.

Es sollte daher auf eine möglichst frühe Intervention und Überleitung in sichere Behandlungspfade gesetzt werden. Die frühere AWMF-Leitlinie zur Lokal-

Die Mitglieder des Expertenrats Strukturentwicklung Wundmanagement werden am Beitragsende gelistet. therapie chronischer Wunden setzt dabei eher am Ende der Therapiekette, nämlich bei der Wundauflage nach abgeschlossener Kausaltherapie an [4].

Vor diesem Hintergrund wurde vom Expertenrat das vorliegende Positionspapier entwickelt und konsentiert. Die Empfehlungen treffen sowohl Aussagen zur Diagnostik als auch zur Therapie über alle medizinischen und nicht medizinischen Behandlungsebenen und -institutionen hinweg. Insbesondere ein einheitliches und zielgerichtetes Handeln aller an der Behandlung von chronischen Wunden Beteiligten sowie eine frühe und schnelle Intervention ist von elementarer Bedeutung für eine rasche Wundheilung und somit ein wesentlicher Einflussfaktor auf die Lebensqualität des Patienten.

\section{》) Rechtzeitige diagnostische Maßnahmen verbessern das Outcome und die Lebensqualität}

Die Diagnostik und Therapie sind unabhängig von der jeweiligen Qualifikation der versorgenden Experten und erfolgen für alle Menschen mit chronischen Wunden nach denselben Prinzipien. Je nach Wissen und Erfahrung sollten behandelnde Ärzte und nichtmedizinische Versorger zur Verbesserung der Lebensqualität des Patienten und mit dem Ziel einer schnellstmöglichen Wundheilung weiteres Expertenwissen hinzuzie- hen. Definierte Diagnostikansätze und Behandlungspfade schaffen eine Übersicht über nötige Einzelschritte, die je nach Erfahrung der behandelnden Ärzte und je nach Indikation in unterschiedlichen Stufen jeweils Diagnoseschritte und Behandlungsziele definieren, die es zu erreichen gilt.

Die beteiligten interprofessionellen und interdisziplinären Kooperationspartner arbeiten in einer offenen Netzwerkstruktur, um zum Wohl der Betroffenen die Versorgung für Menschen mit chronischen Wunden ausreichend, zweckmäßig und wirtschaftlich auszurichten. Dies hilft Versorgungsbrüche, Unter-, Über- und Fehlversorgungen zu vermeiden.

\section{Methode}

In 3 Arbeitsgruppen (Diagnostik, Therapie und Versorgungsstruktur) wurden auf der Grundlage von Expertenwissen die Schritte für eine notwendige Frühintervention und die Weiterleitung in eine geordnete und strukturierte Wundversorgung entwickelt.

In mehreren Workshops wurden die Ergebnisse der Arbeitsgruppen anschließend diskutiert und konsentiert.

\section{Qualifikation}

Der Expertenrat legt Mindestqualifikationsstandards für Handelnde in der chro- 


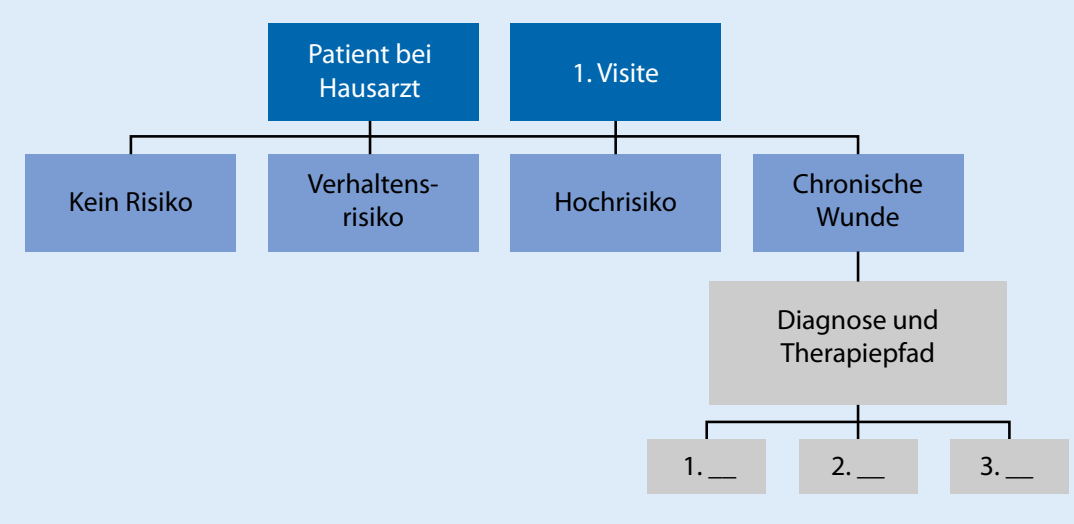

Abb. 1 \& Schema zu Prävention und Therapie chronischer Wunden

nischen Wundversorgung zugrunde. Der Nachweis erfolgt durch Fortbildung oder spezifisches Erfahrungswissen in der Behandlung chronischer Wunden. Als Basis dient dabei ein von Prof. Dr. Augustin für den Bundesverband der $\mathrm{AOK}$ entwickelter Ansatz, der aber weiterentwickelt wurde. Dieses erweiterte Konzept wird am Ende des Artikels dargestellt (॰ Abb. 6).

Zur speziellen Einbindung und Prävention von Risikopatienten wird folgendes Vorgehen ( $\bullet$ Abb. 1) empfohlen.

Anhand des Schemas haben die Arbeitsgruppen die weiteren Schritte für eine notwendige Frühintervention und die Weiterleitung in eine geordnete und strukturierte Wundversorgung ausformuliert.

\section{Diagnostik}

Die ärztliche Basisdiagnostik, die weiterführende Diagnostik und die unterschiedlichen Indikationen und Level der Behandlungsschritte sind wesentliche Eckpunkte einer adäquaten Versorgung chronischer Wunden. Beschrieben werden in diesem Papier dabei die Einzelschritte der Diagnostik nach bestehenden Standards. Sollte ein behandelnder Arzt nicht über bestimmte Diagnostikmöglichkeiten verfügen, so ist anzustreben, den Diagnoseschritt durch entsprechende Netzwerkpartner zu erreichen. Damit dies gewährleistet ist, bedarf es einer engmaschigen Verlaufskontrolle bis zum Erreichen einer vollständigen Diagnose. Erst danach ist es möglich, einen der
Situation angemessenen Therapieplan zu erstellen (• Abb. 2).

\section{Therapie}

Die anzuwendenden Therapien für unterschiedliche Indikationen und Diagnosen sowie die eingebundenen Versorgungspartner wurden durch die Arbeitsgruppe Therapie bearbeitet und strukturiert dargestellt ( $\bullet$ Abb. 3, 4 und 5). Als Orientierung hat die Expertengruppe zusätzlich Anforderungen an einen Mindestqualifikationsstandard der Therapieakteure aufgeführt.

\section{Empfehlung: Versorgungs- struktur in Form eines Netzwerks}

Die Arbeitsgruppe Versorgungsstruktur empfiehlt unbedingt den Aufbau eines Versorgungsnetzwerks für die Behandlung von Patienten mit chronischen Wunden.

Im Folgenden wurden drei Thesen formuliert, die für die Schaffung eines interprofessionellen Netzwerks von elementarer Bedeutung sind.

\section{Thesen einer interprofessionellen Arbeitsgruppe im Rahmen der jeweiligen Versorgungsstruktur}

1. Keine Berufsgruppe kann eine adäquate Wundversorgung chronischer Wunden alleine bewältigen. Die Herausforderungen für eine interprofessionelle, interdisziplinäre und transsektorale Versorgung im Bereich von chronischen und/oder palliativen Wunden sind groß.

2. Grundlage für die verschiedenen Phasen der Versorgung ist ein partnerschaftliches Miteinander auf Augenhöhe unter Beachtung und Anerkennung der gegenseitigen Kompetenzen.

3. Es sollte ein Ablauf angestrebt werden, der für alle beteiligten Berufsgruppen transparent, kontinuierlich und qualitätsgesichert ist. So können sich alle Beteiligten auf die Vorarbeiten der anderen Berufsgruppen verlassen und auf diesen aufbauen.

\section{Aufgaben und Zielsetzung des Netzwerks}

- Das Netzwerk sichert die interprofessionelle, interdisziplinäre und transsektorale Versorgung im Bereich von chronischen Wunden und/oder palliativer Wundbehandlung.

- Vernetzte Versorgung vermeidet Unter-, Über- und Fehlversorgung.

- In diesem Netzwerk arbeiten die Versorgungsbereiche und die an der Versorgung beteiligten Berufsgruppen transparent, kontinuierlich und qualitätsgesichert zusammen. Die Zusammenarbeit erfolgt schnittstellenübergreifend.

- Die am Netzwerk Beteiligten erarbeiten zunächst die in der Region notwendigen Struktur- und Prozesskriterien, um dann gemeinsam die Aufgaben, die Verantwortlichkeiten und den Weg der Kommunikation zu definieren.

- Zur Selbstorganisation benennt das Netzwerk eine Netzwerkkoordination.

- Das Netzwerk entwickelt Standards für alle erforderlichen Prozessschritte für eine hochwertige Versorgung von chronischen Wunden.

- Das Netzwerk stellt sicher, dass ein Lotse für jeden Wundpatienten zur Verfügung steht. Aufgabe des Lotsen ist die Koordination der Versorgung und der beteiligten Netzwerkpartner.

- Jeder Netzwerkpartner ist eigenverantwortlich und verpflichtet sich, die Versorgung leitliniengerecht und in der geforderten Sorgfalt, Voll- 
ständigkeit, Richtigkeit und Qualität zeitgerecht durchzuführen.

- Die umfassende Dokumentation aller durchgeführten Schritte ist verpflichtend.

- Das Dokumentationssystem sollte in der jeweiligen Region aufeinander abgestimmt werden.

- Das Netzwerk schafft eine gemeinsame Kommunikationsplattform, um einen routinemäßigen, niedrigschwelligen und strukturierten Informationsaustausch zwischen den Beteiligten zu ermöglichen.

- Das Netzwerk führt regelmäßig Netzwerktreffen, Fallkonferenzen und Qualitätszirkel durch.

\section{Empfehlungen für den \\ Netzwerkaufbau und die Umsetzung der Aufgaben}

- Die Berufsgruppen erarbeiten vor Ort (z.B. in der ambulanten Versorgung, in der stationären Pflegeeinrichtung, im Krankenhaus) gemeinsam eine Verteilung von Aufgaben und Verantwortlichkeiten, damit Synergieeffekte maximal genutzt werden können.

- Eine Standardisierung aller erforderlichen Prozessschritte garantiert dabei ein hohes Maß an Sicherheit und Qualität.

- Die umfassende Dokumentation aller durchgeführten Schritte ist essenziell, um eine maximale Transparenz und Reproduzierbarkeit für alle Beteiligten und für Dritte zu gewährleisten.

- Alle beteiligten Versorgungsteams übernehmen die Verantwortung für die von ihnen durchgeführten Prozessschritte auf dem aktuellen Stand des medizinischen Wissens, d.h. sie tragen dafür Sorge, dass diese in der geforderten Sorgfalt, Vollständigkeit, Richtigkeit und Qualität zeitgerecht durchgeführt werden.

- Eine Vereinheitlichung der verwendeten Begrifflichkeiten fördert die Kommunikation zwischen den Berufsgruppen und gewährleistet ein einheitliches Verständnis und eine standardisierte Dokumentation der Prozessschritte.

Gefässchirurgie 2019·24:388-398 https://doi.org/10.1007/s00772-019-0550-9

๑) Springer Medizin Verlag GmbH, ein Teil von Springer Nature 2019

\section{Storck · J. Dissemond · V. Gerber · M. Augustin · Expertenrat Strukturentwicklung Wundmanagement \\ Kompetenzlevel in der Wundbehandlung. Empfehlungen zur Verbesserung der Versorgungsstruktur für Menschen mit chronischen Wunden in Deutschland}

\section{Zusammenfassung}

Menschen mit chronischen Wunden sehen sich vielfältigen wund- und therapiebedingten Einschränkungen im Alltagsleben ausgesetzt. Verbunden sind damit neben körperlichen Beeinträchtigungen (z. B. Schmerzen, Geruch und Exsudatmengen) auch Einschränkungen der Selbstständigkeit, des sozialen Lebens und Einschränkungen in Bezug auf ihr Selbstbild. In Deutschland existieren keine Empfehlungen zur Vereinheitlichung einer rechtzeitigen Diagnostik und Therapie, mit dem Ziel einer frühen Intervention. Es wurde daher eine Expertengruppe aus verschiedenen Sektoren des Gesundheitswesens gegründet, um einen Konsensus zu formulieren. In den Empfehlungen sind die interprofessionellen Zuständigkeiten sowie ein Zeitpunkt einer rechtzeitigen Einbeziehung von Fachärzten/spezialisierten Wundzentren definiert. Durch Implementation der erarbeiteten Empfehlungen in das bestehende System kann die Versorgungsqualität von Patienten mit chronischen Wunden flächendeckend verbessert werden.

\section{Schlüsselwörter}

Chronische Wunden - Versorgungsqualität · Frühintervention · Wundzentren . Expertenkonsens

\section{Competence levels in wound care. Recommendations for improvement of care structures for patients with chronic wounds in Germany}

\section{Abstract}

Patients with chronic wounds are highly limited with respect to the activities of daily life due to many wound and treatmentrelated aspects. Among those limitations are physical impairments (e.g. pain, amount of exudate and odor) and impairments of self-sufficiency and social life including a distortion of the self-image. In Germany, no uniform recommendations exist with respect to early and sufficient diagnostics and treatment of chronic wounds, with the specific aim of an early intervention. Therefore, a multidisciplinary expert group including healthcare providers, insurance companies and a member of the Ministry of Health was constituted to elaborate a consensus statement. In the recommendations the interprofessional responsibilities and the timing of inclusion of specialised physicians and specialized wound centers for the care of such patients are defined. The quality of care of patients with chronic wounds can be improved if the suggested recommendations are comprehensively implemented in the existing healthcare system.

\section{Keywords}

Chronic wounds - Quality of health care Early Intervention - Wound centers - Expert consensus
- Eine gemeinsame Kommunikationsplattform ist unverzichtbar, um einen routinemäßigen, niedrigschwelligen und strukturierten Informationsaustausch zwischen den Beteiligten, z. B. Ärzte und Fachärzte, Medizinische Fachangestellte, Pflegefachkräfte und Patienten/Angehörige zu ermöglichen.

- Es sollte jede Möglichkeit genutzt werden, den persönlichen Austausch zwischen den Berufsgruppen zu fördern, z. B. durch interdisziplinäre Visiten oder Qualitätszirkel.

- Das Netzwerk gibt sich ein Leitbild und eine Geschäftsordnung.

- Die Netzwerkbildung, die Netzwerkteilnahme und die Zusammenarbeit im Netzwerk müssen mit Interessenvertretern nach rechtlichen Grundlagen erfolgen. Wirtschaftsunternehmen achten die Health Care Compliance. 


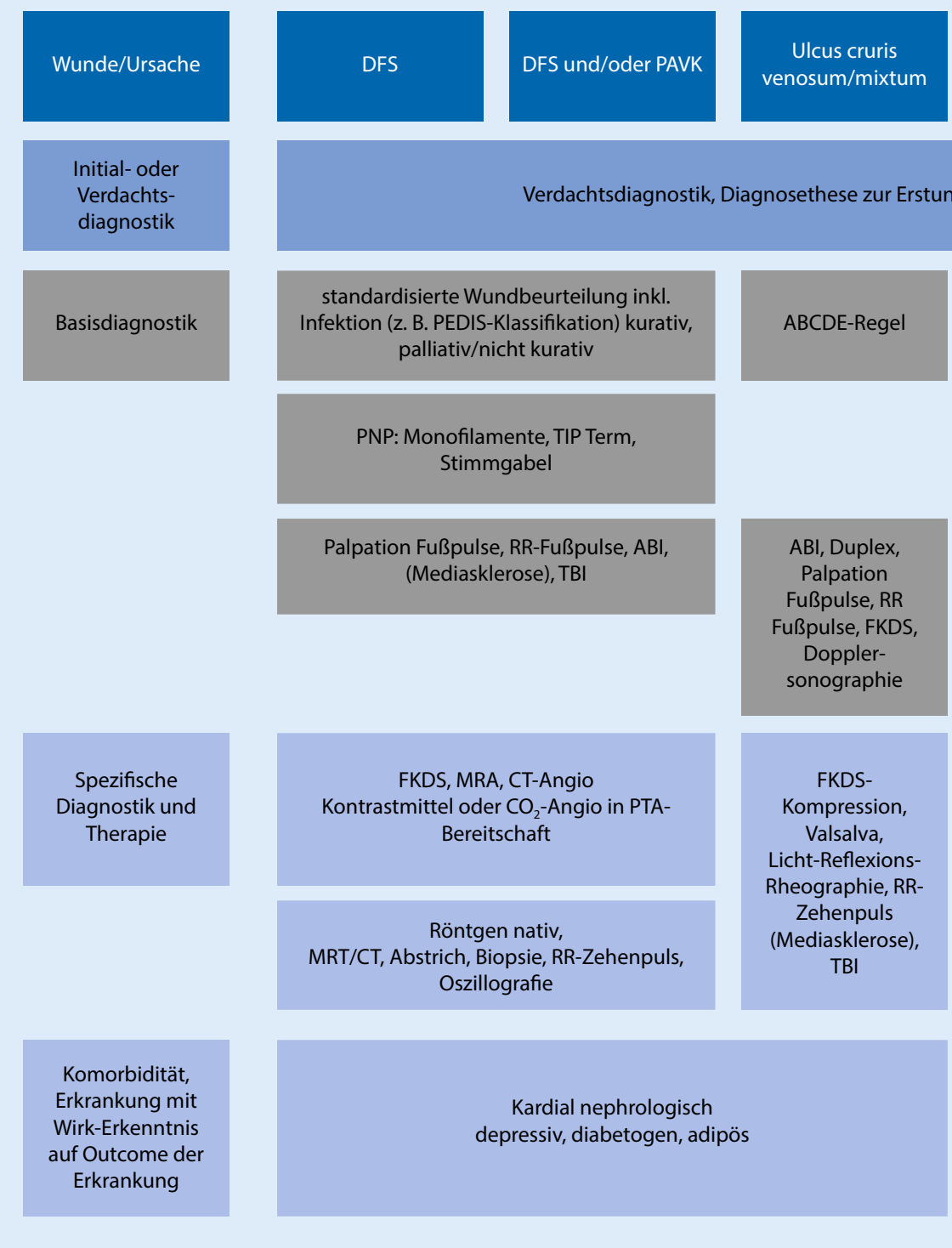

Exulzerierende

Tumoren/palliative

Wunden

(Wunden

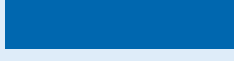

nitial- oder

Verdachts-

diagnostik

Verdachtsdiagnostik, Diagnosethese zur Erstuntersuchung/Anamnese

standardisierte Wundbeurteilung inkl.
Infektion (z. B. PEDIS-Klassifikation) kurativ,
palliativ/nicht kurativ

PNP: Monofilamente, TIP Term, Stimmgabe

Fußpulse, RR-Fußpulse, $A B I$ (Mediasklerose), TB

Thostik und Therapie

Komorbidität, Erkrankung mit Wirk-Erkenntnis Uf Outcome de Erkrankung

Abb. $2 \Delta$ Diagnostikschritte für chronische Wunden für verschiedene Indikationen

\section{Netzwerkpartner: Eignung und Qualifikation}

Die folgenden Empfehlungen legen dar, über welche notwendigen Qualifikationen die einzelnen Partner verfügen sollten. Grundlage dazu ist die interprofessionelle, interdisziplinäre und transsektorale Zusammenarbeit in einem kooperierenden Verbund unterschiedlicher Berufsgruppen und Institutionen/ Strukturen. Darüber hinaus geben die Experten Hinweise darauf, welche Ausstattung die eingebundenen Partner für die Versorgung chronischer Wunden mitbringen sollten.

\section{Einrichtungen}

Arztpraxis: Hausarzt/allgemein hausärztliche Versorgung, praktischer Arzt, Arzt für Allgemeinmedizin, Internist ohne Schwerpunkt, Kinder- und Jugendmediziner, Palliativmediziner:

- Behandlungserfahrung im Umgang mit Menschen mit chronischen Wunden unterschiedlicher Genese und/oder palliativen Wunden

- Basiswissen über Diagnostik und Behandlung entsprechend der medizinischen Leitlinien:

- Daraus Ableitung der Entscheidung, bis zu welchen Diagnostikund Behandlungsschritten diese vom Arzt selbst durchgeführt wer-
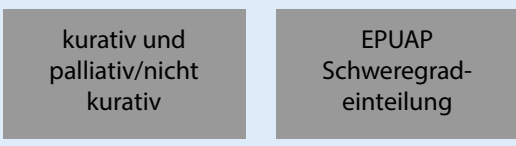

Statuserhebung

Primärer Fokus

Leidlinderung
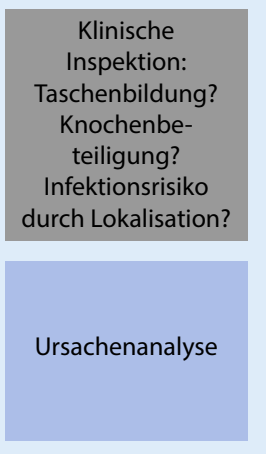

Lebensbegrenzende Erkrankungen (Tage, Wochen, Monate)
Bewegungseinschränkungen, Spastiken,

Lähmungen,

Sensibilitätsstörungen den, wann sie nötig sind und ab welchem Schritt weitere Fachdisziplinen hinzugezogen werden

- Behandlung:

- Beherrschung verschiedener Débridement-Techniken

- Auswahl der adäquaten, medizinisch notwendigen Wundtherapie

- Erkennung und Beherrschung von Wundinfektionen

- Kompressionsanwendung und -techniken einleiten

- Hilfsmittelversorgung

- MFA/Pflegefachkräfte:

- Kompressionsanwendung und -techniken beherrschen 


\section{Leitthema}
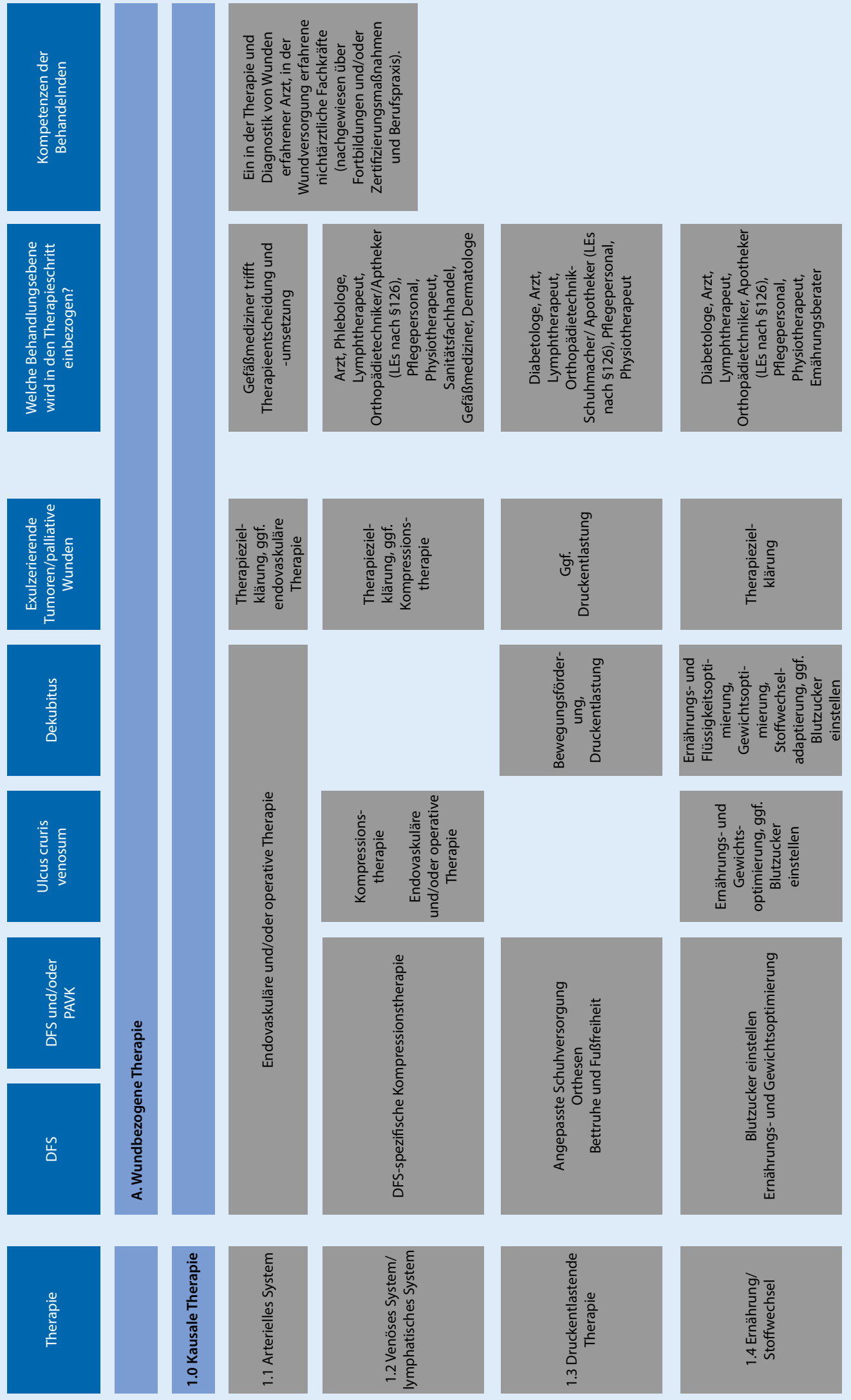

Abb. $3<$ Ziele und therapeutische Maßnahmen für verschiedene Indikationen einschließlich der Behandlungsebenen und Kompetenzen (Teil 1). DFS Diabetisches Fußsyndrom 

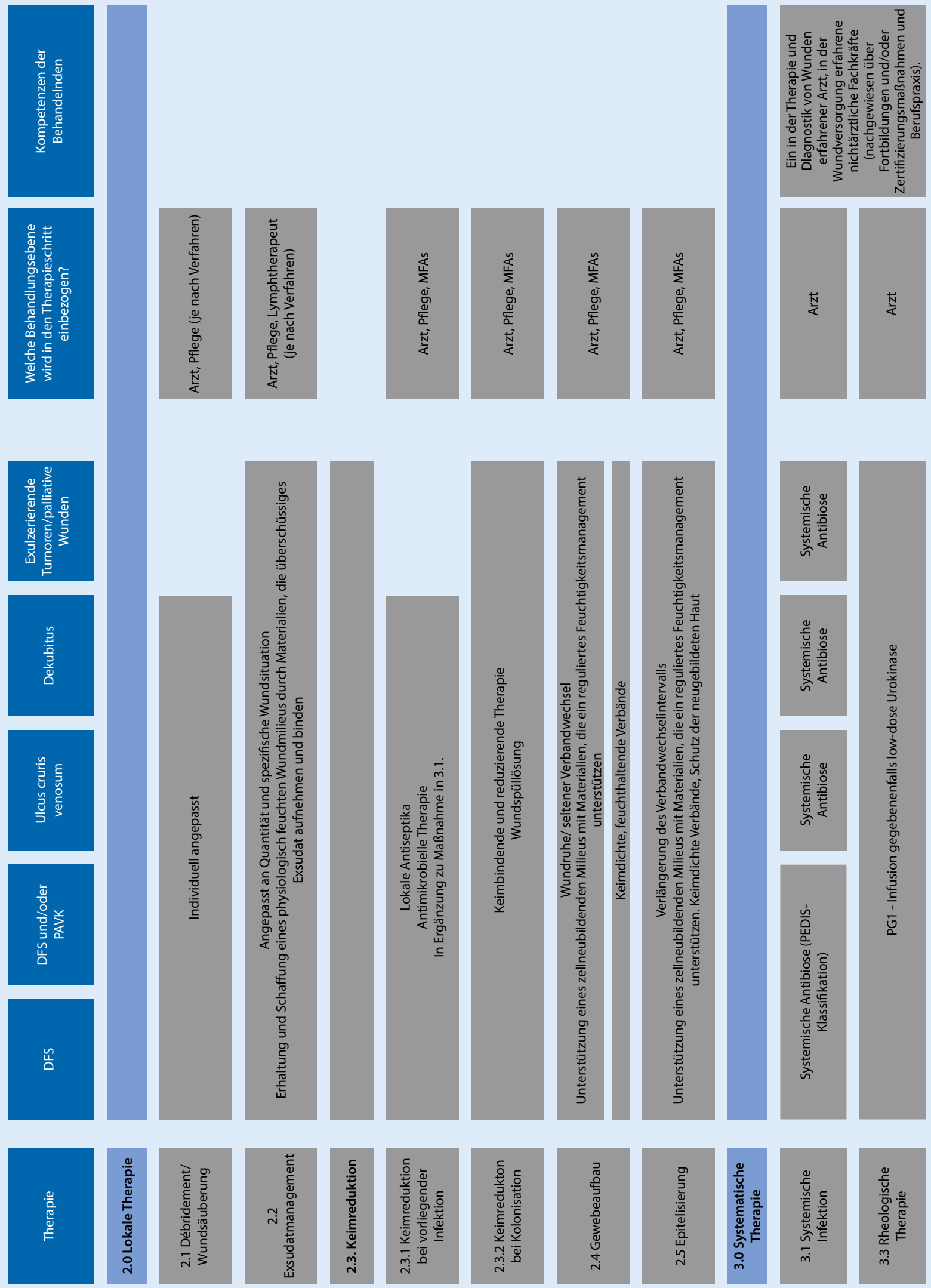

Abb. $4 \Delta$ Ziele und therapeutische Maßnahmen für verschiedene Indikationen einschließlich der Behandlungsebenen und Kompetenzen (Teil 2) 


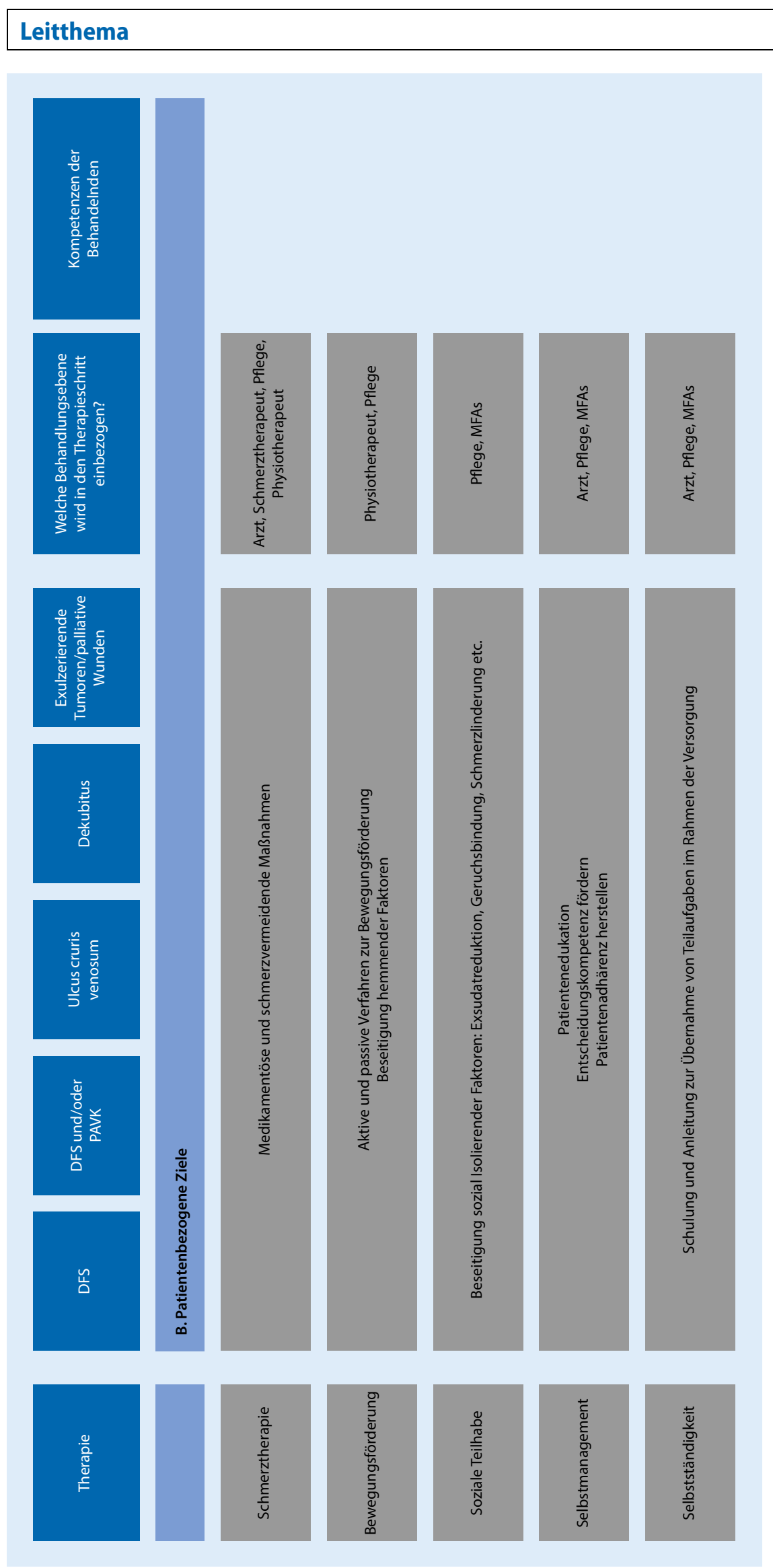

Abb. $5 \Delta$ Ziele und therapeutische Maßnahmen für verschiedene Indikationen einschließlich der Behandlungsebenen und Kompetenzen (Teil 3).DFS Diabetisches Fußsyndrom, PAVK periphere arterielle Verschlusskrankheit
- Wünschenswert: Zusatzqualifikation Wundversorgung

- Nichtärztliche Praxisassistenz (NäPa) als Schnittstelle in der Versorgung sinnvoll

Spezialisten in alphabetischer Reihenfolge, vorausgesetzt werden Erfahrung in der Behandlung von chronischen Wunden sowie Basiswissen über Diagnostik und Behandlung:

- Allgemeinmediziner mit spezieller wundärztlicher Ausrichtung

- Angiologen

- Chirurgen/Fußchirurgen (DFS)

- Dermatologen

- Diabetologen:

- DDG-Zusatzqualifikation diabetisches Fußsyndrom - als anerkannte Schwerpunktpraxis/ Fußambulanz/Klinikabteilung, Anforderungsmerkmale der AGFuß der Deutschen Diabetologischen Gesellschaft

- Teilnahme am Disease-Management-Programm „Diabetes“

- Kenntnis der Grundlagen der Druckentlastung, des „Filzens“ und der Wundtherapie (sog. „Filzen“ dient der Druckentlastung beim diabetischen Fußsyndrom)

- Gefäßchirurgen

- Orthopäden/technische Orthopäden

- Palliativmediziner

- Phlebologen

- Plastische Chirurgen/Wiederherstellungschirurgen

- Schmerztherapeuten

- Eingebundenes Fachpersonal in den Einrichtungen (wünschenswert: Behandlungserfahrung von chronischen Wunden, Basiswissen über Prävention, Wund- und Kompressionstherapie):

- MFA

- Pflegefachkräfte

- Physician Assistants

\section{Spezielle Einrichtungen und weitere Anlaufstellen}

Ambulante Pflegeeinrichtungen (inkl. spezieller Palliativpflege):

- Unter anderem Einsatz von qualifiziertem Pflegefachpersonal zur Wundversorgung, EDV zur Wunddokumentation, Qualitätsmanagement- 
system für Behandlung chronischer Wunden

- Umsetzung und Einhaltung der Expertenstandards bzw. zertifiziert, zum Beispiel von der Initiative Chronische Wunden e. V. (ICW), Deutsche Gesellschaft für Wundversorgung e. V. (DGfW), der Akademie Kammerlander, Deutsche Dermatologische Gesellschaft (DDG) oder analoge Standards

- Pflegefachkräfte:

- Beherrschung von Kompressionsanwendung und -techniken

- Beratungs- und edukative Kompetenz

- Pflegefachkräfte mit Zusatzqualifikation:

- Zum Beispiel zertifiziert von ICW, DGfW, Kammerlander, DDG oder analoge Standards

- Beratungs- und edukative Kompetenz

Stationäre Pflegeeinrichtungen:

- Unter anderem Einsatz von qualifiziertem Pflegefachpersonal zur Wundversorgung, EDV zur Wunddokumentation, Qualitätsmanagementsystem für Behandlung chronischer Wunden

Stationäre Hospize.

Spezialisierte ambulante Palliativversorgung (SAPV). fe.

Einrichtungen der Eingliederungshil-

\section{Bedarfsorientierte, ergänzende Professionen}

Podologen:

- Spezielle Kenntnisse zur Prävention, Lokaltherapie, Druckentlastung und zu Risiken beim diabetischen Fuß

Physiotherapeuten:

- Spezielle Kenntnisse zur Ödemtherapie

- Manuelle Lymphtherapie

Ernährungsberatung:

- Schwerpunkt Diabetes

Sonstige Leistungserbringer nach $₫ 126$ SGB V
Kenntnisse zur Anwendung und Anleitung für Produkte zur Wundversorgung und Zusatzschulungen für spezifische Hilfsmittel (Kompression, Lagerung, Anti-Dekubitus, Gehhilfen etc.):

- Orthopädische Fachgeschäfte

- Apotheken

- Homecare/Sanitätshaus

- Orthopädieschuhmacher:

- DFS-ausgerichtet

- Spezielle Schuhzurichtung wie TCC, Orthesen, Diabetic-Walker

\section{Netzwerkstrukturen}

Das Netzwerk zur Behandlung chronischer Wunden sollte einheitlichen Strukturen folgen. Die Erfordernisse orientieren sich dabei an ICW-Wundsiegelkriterien, der Zertifizierung AG diabetischer Fuß DDG und der Akademie Kammerlander.

Strukturerfordernisse, orientiert an ICW-Wundsiegelkriterien, Zertifizierung AG diabetischer Fuß DDG, Akademie Kammerlander:

\section{Einrichtungen}

Arztpraxis:

- Praxisausstattung zur Wundversorgung: Hygiene, Diagnostik, Behandlungsraum

- EDV-System zur Wunddokumentation

- Telematik-Infrastruktur

- Qualitätsmanagementsystem für Behandlung chronischer Wunden

Klinik/Krankenhaus:

- Krankenhausinternes Wundmanagement (zum Beispiel Wundkonsildienst mit zugeordneten Wundexperten bzw. qualifizierten Ärzten und Pflegefachkräften in der Wundversorgung) - dezentral/zentral organisiert

- Entlassmanagement mit Wundmanagement

- EDV-System zur Wunddokumentation

- Perspektivisch: regelhaft telemedizinische Technik und Voraussetzungen

- Telematik-Infrastruktur

- Qualitätsmanagementsystem für Behandlung chronischer Wunden in Anlehnung an die ICW-Wundsiegelkriterien
- Realisation der nationalen Expertenstandards (Dekubitus/chronische Wunden/Schmerz) und der entsprechenden Leitlinien

Ambulante Pflegeeinrichtungen: zum Beispiel zertifiziert von ICW, DGfW, Kammerlander, DDG oder analoge Standards.

Spezialisierte Einrichtungen zur Versorgung chronischer Wunden:

- Zum Beispiel Wundzentrum, Wundambulanz, spez. Pflegedienste etc. zum Beispiel zertifiziert von ICW, DGfW, Kammerlander, DDG oder analoge Standards

\section{Fazit für die Praxis}

Ziel der vorgelegten Empfehlungen ist es, die Versorgung von Menschen mit chronischen Wunden zu verbessern. Ausgehend von der These, dass eine möglichst frühe Intervention bei den Patienten zu einer schnelleren Diagnose und Therapie führt, die ihre Situation erleichtert, haben die beteiligten Experten die Empfehlungen als möglichen Rahmen für eine zukünftige Struktur zur Wundversorgung formuliert. In einem weiteren Schritt wird angestrebt, in möglichst breitem Konsens weitere Maßnahmen zur Versorgungsverbesserung auf Basis dieser Empfehlungen breit zu diskutieren.

Als Übersicht wurde die Darstellung der Zielsetzung für eine optimierte Versorgung chronischer Wunden entsprechend den vorgenommenen Ergänzungen modifiziert. Anstelle der ursprünglich dargestellten Therapieziele wurden die beteiligten interprofessionellen und interdisziplinären Kooperationspartner integriert.

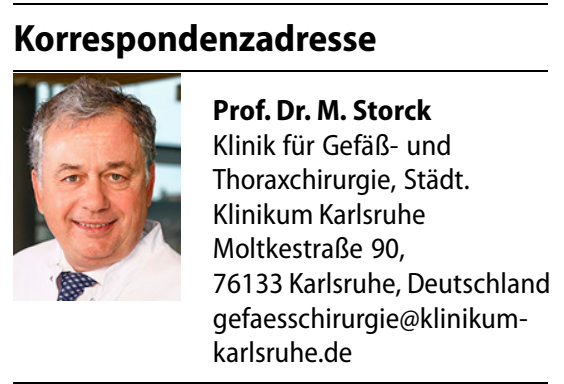




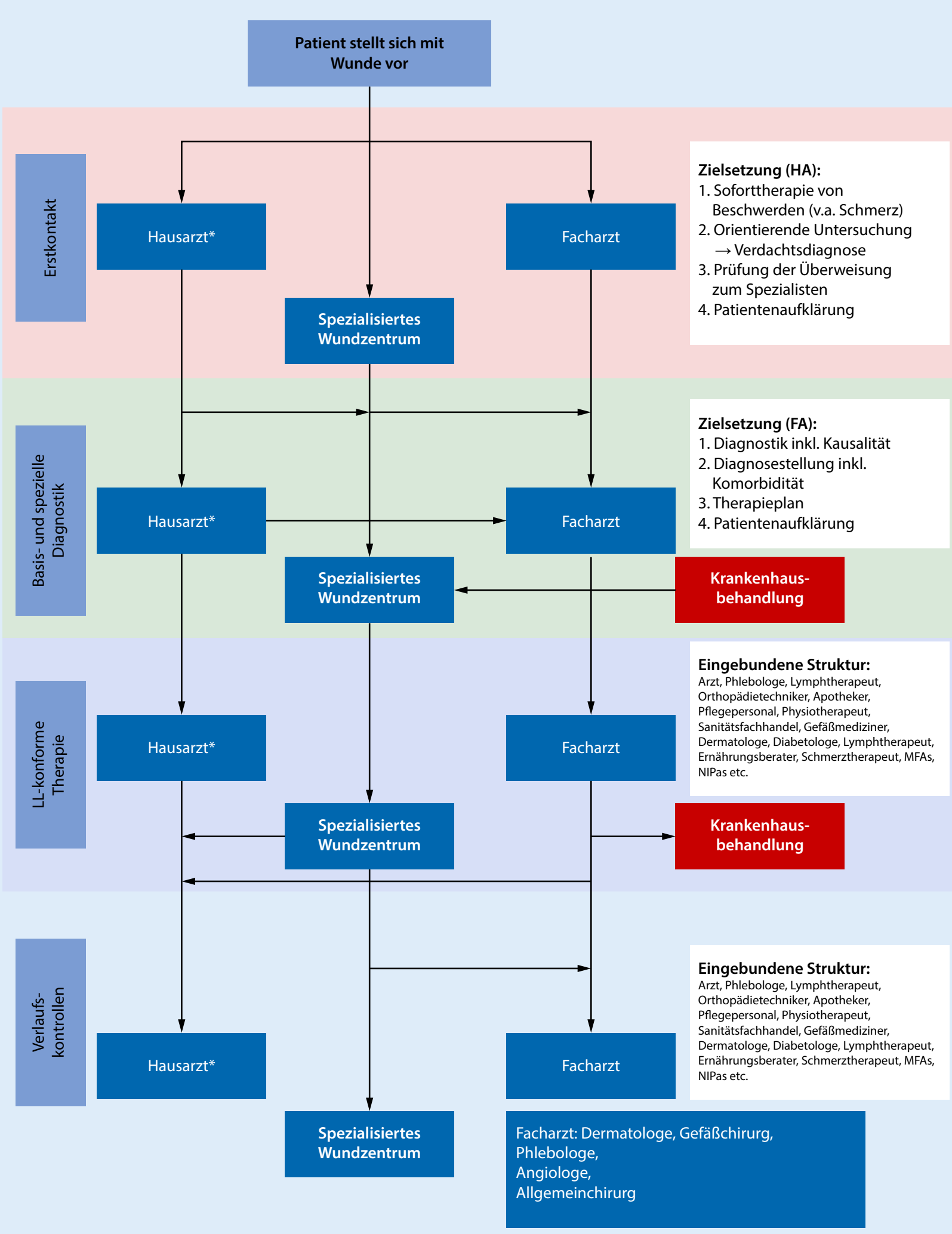

Abb. 6 \ Weiterentwickeltes Schema zur optimierten Versorgung chronischer Wunden (hier: Indikation Ulcus cruris). (Mod. nach Augustin, M., AOK-BV Abschlussbericht, pers. Mitteilung). HA Hausarzt, FA Facharzt 


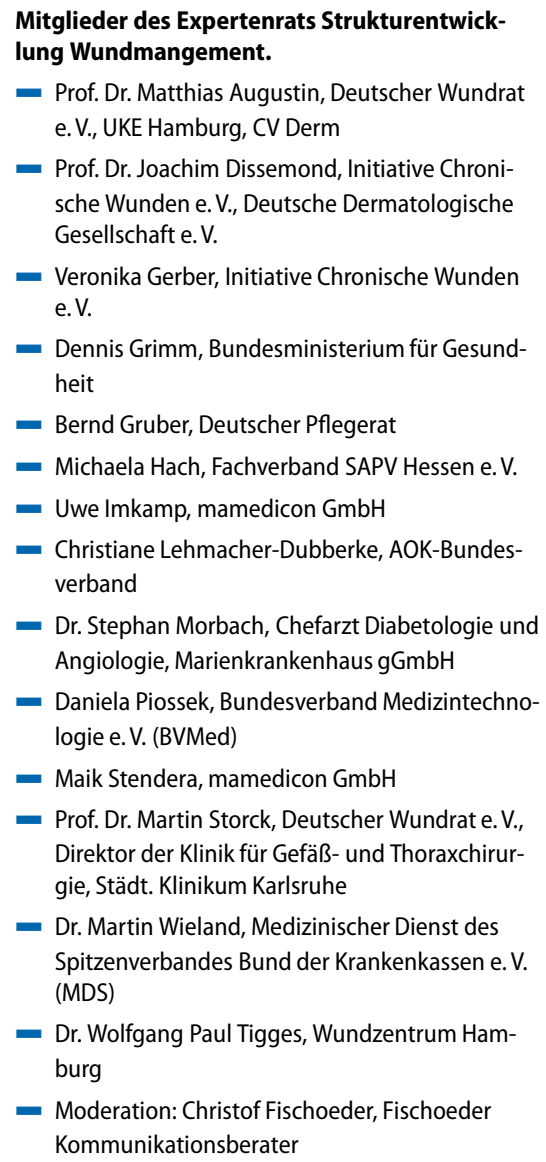

\section{Einhaltung ethischer Richtlinien}

Interessenkonflikt. M.Storck, J.Dissemond, V.Gerber und M. Augustin geben an, dass kein Interessenkonflikt besteht.

Für diesen Beitrag wurden von den Autoren keine Studien an Menschen oder Tieren durchgeführt. Für die aufgeführten Studien gelten die jeweils dort angegebenen ethischen Richtlinien.

\section{Anhang}

\section{Erläuterungen zu benutzten Abkürzungen/Glossar}

\section{Wunde}

Als Wunde wird der Barriereverlust zwischen dem Körper und der Umgebung durch Zerstörung von Gewebe an äußeren oder inneren Körperoberflächen bezeichnet.

\section{Chronische Wunde}

Eine Wunde, die nach 8 Wochen nicht abgeheilt ist, wird als chronisch bezeichnet.
Unabhängig von dieser zeitlich orientierten Definition gibt es Wunden, die von Beginn an als chronisch anzusehen sind, da Ihre Behandlung eine Therapie der weiterhin bestehenden Ursache erfordert. Hierzu gehören beispielsweise das diabetische Fußulkus, Wunden bei PAVK, Ulcus cruris venosum oder Dekubitus.

\section{Wundexsudat}

Als Wundexsudat werden alle Flüssigkeiten bezeichnet, die von einer Wunde freigesetzt werden. In Abhängigkeit des Wundzustands kann diese Lymphe, Blut, Proteine, Keime, Zellen und Zellreste beinhalten [5].

\section{ABCDE-Regel der Diagnostik chronischer Wunden}

- A - Anamnese

- B - Bakterien

- C - Klinische Untersuchung

- D - Durchblutung

- E-Extras [6]

\section{Débridement}

Arten des Débridements (nach EWMA [European Wound Management Association]) [7]

- Mechanisches Débridement, z. B. mit sterilen Kompressen

- Scharfes Débridement, z.B. mit „scharfem Löffel“

- Chirurgisches Débridement, z. B. mit Dermatom

- Autolytisches Débridement, z. B. mit Hydrogel

- Enzymatisches Débridement, z. B. mit Kollagenasen

- Jet Lavage/Hydrochirurgie, z. B. mit hochdruckbeschleunigter Ringerlösung

- Ultraschall-Débridement, z. B. mit niederfrequentem Leistungsultraschall

- Madentherapie, z. B. mit Fliegenmaden der Gattung Lucillia sericata

\section{DFS}

Diabetisches Fußsyndrom.

\section{EPUAP}

European Pressure Ulcer Advisory Panel.

\section{PEDIS-Klassifikation}

Die PEDIS-Klassifikation ist eine Facetten-Klassifikation zur Einteilung des diabetischen Fußsyndroms.

\section{Aufbau}

In der PEDIS-Klassifikation wird eine Läsion nach folgendem Schema erfasst [8]:

- Perfusion:

- Grad 1: Keine Symptome oder klinischen Zeichen einer PAVK

- Grad 2: Symptome einer PAVK ohne kritische Ischämie

- Grad 3: Kritische Ischämie

- Extent: Fläche in $\mathrm{cm}^{2}$

- Depth: Gewebedefekt:

- Grad 1: Oberflächliches Hautulkus/ Erosion

- Grad 2: Ulkus, das durch die Dermis in tiefe Hautschichten reicht

- Grad 3: Tief reichendes Ulkus (Weichteile, Sehnen, Knorpel, Knochen)

- Infektion:

- Grad 1: Keine Symptome oder klinischen Zeichen

- Grad 2: Entzündung der Haut oder des Subkutangewebes

- Grad 3: Ausgedehntes Erythem, das in tiefe Gewebeschichten reicht

- Grad 4: SIRS (Systemic inflammatory response syndrome)

- Sensation (Empfindungsstörung):

- Grad 1: Kein Verlust der protektiven Sensibilität

- Grad 2: Protektive Sensibilität eingeschränkt (z.B. bei diabetischer Polyneuropathie)

\section{Literatur}

1. Heyer K, Herberger K, Protz K, Glaeske G, Augustin M (2016) Epidemiology of chronic wounds in Germany: Analysis of statutory health insurance data. Wound Repair Regen 24:434-442

2. Diener H, Debus E, Herberger K, Heyer K, Augustin M, Tigges W, Karl T, Storck M (2017) Versorgungssituation gefäßmedizinischer Wunden in Deutschland. Gefasschirurgie 22:548-557

3. Augustin M, Schmitt J, Herberger K et al (2014) The German national consensus on wound documentation and outcomes: Rationale, working programme and current status. Wound Med 7:8-13

4. Rüttermann $M$, Maier-Hasselmann A, NinkGrebe B, Burckhard M (2013) Local treatment of chronic wounds in patients with peripheral vascular disease, chronic venous insufficiency and diabetes. Dtsch Arztebl Int 110:25-31 
5. Dissemond J, Bültemann A, Gerber V, Jäger B, Münter C, Kröger K (2016) Definitionen für die Wundbehandlung. Hautarzt 67:265-266

6. Dissemond J (2017) ABCDE-Regel der Diagnostik chronischer Wunden. J Dtsch Dermatol Ges 15:732-734

7. Strohal R, Dissemond J, Jordan O'Brien J, Piaggesi A, Rimdeika R, Young T, Apelqvist J (2013) EWMA Dokument 2013: Debridement. Stand der Entwicklung und Rolle des Debridements: Aktuelle Übersicht und Begriffserklärungen. Wundmanagement Suppl. 3:4-39

8. https://flexikon.doccheck.com/de/PEDISKlassifikation.Zugegriffen:07.08.2019

Kontinuumsroboter - Prototypen demonstrieren die Beweglichkeit

Biegsame Roboterarme: für große Greifer und kleine Endoskope

Sie können sich präzise um Windungen und Ecken schlängeln, bewegen sich frei in alle Richtungen: Biegsame Roboterarme, die Prof. Stefan Seelecke und seine Forschergruppe an der Universität des Saarlandes entwickeln, haben keine steifen Gelenke, dafür aber Muskeln aus Formgedächtnis-Drähten. Diese brauchen weder Druckluft noch schweres Zubehör, sondern funktionieren nur mit elektrischem Strom. Das Material selbst hat Sensoreigenschaften, daher lassen sich die Arme ohne zusätzliche Sensoren steuern. Große Roboter-Rüssel können mit der neuen Technologie ebenso ausgestattet werden wie haarfeine Tentakel für endoskopische Operationen.

\section{Präzise steuerbare Tentakel}

In einem von der Deutschen Forschungsgemeinschaft geförderten Projekt arbeitet das Forscherteam gemeinsam mit Kollegen der TU Darmstadt an dünnen, präzise steuerbaren künstlichen Tentakeln: Diese können in Zukunft in der Medizin als Führungsdraht bei Herzoperationen oder als Endoskop bei Magen- und Darmspiegelungen zum Einsatz kommen. Dafür werden die Tentakel mit zusätzlichen Funktionen ausgestattet, etwa einer versteifbaren Spitze für Stoßbewegungen oder einem Greifer. Im großen Stil funktioniert die Technologie ebenso: Auch große Roboter-Rüssel sind möglich - die Technik ist skalierbar.

\section{Künstliche Muskelstränge}

Dreh- und Angelpunkt sind die künstlichen Muskeln: Die Muskelstränge bestehen aus haarfeinen Drähten aus Nickel-Titan, die anspannen und entspannen können. Sie kontrahieren wie echte Muskeln, je nachdem ob Strom fließt oder nicht. Die Legierung $\mathrm{Ni}$ ckel-Titan besitzt ein so genanntes Formgedächtnis. Wird ein Draht aus diesem Material verbogen, kann er seine ursprüngliche Form wieder annehmen. Fließt Strom durch einen solchen Draht, erwärmt er sich und seine Kristallstruktur wandelt sich so um, dass er sich verkürzt. Wird der Strom abgeschaltet, kühlt er ab und wird wieder lang.
Die feinen Drähte werden gebündelt wie Muskelfasern. Mehrere Drähte geben durch die größere Oberfläche mehr Wärme ab, dadurch werden schnelle Kontraktionen erreicht. Die Drähte haben nach Angaben von Seelecke die höchste Energiedichte aller bekannten Antriebsmechanismen. Auf kleinem Raum entwickeln sie hohe Zugkraft.

\section{Katheter oder Endoskop}

Bei ihren Roboter-Armen verbinden die Ingenieure die Drahtstränge als Beuge- und Streck-Muskulatur, so dass ihr Zusammenspiel eine fließende Bewegung hervorbringt. Bei der Tentakel, die in der Medizin künftig etwa als Katheter oder Endoskop Anwendung finden kann, kommt man hierbei mit einem Durchmesser von etwa 300 bis 400 Mikrometer aus. Auf diesem Raum lassen sich sonst keine anderen Antriebstechniken unterbringen, was etwa die Möglichkeiten bisheriger Katheter-Verfahren einschränkt, erläutert das Team.

\section{Drähte haben Sensoreigenschaften}

Im Gegensatz dazu ist die Tentakel hochpräzise steuerbar und kann als Werkzeug mehrere Funktionen erfüllen, etwa mit ihrer Spitze stoßen. Die Forscher modellieren und programmieren hierzu Bewegungsmuster zur Steuerung auf einen Halbleiterchip. Das System kommt dabei völlig ohne Sensoren aus. Die Drähte liefern selbst alle nötigen Daten. Das Material der Drähte hat Sensoreigenschaften. Die Steuerungseinheit erkennt anhand der Messdaten des elektrischen Widerstandes zu jeder Zeit die genaue Position und Ausrichtung der Drähte.

Quelle: Universität des Saarlandes www.uni-saarland.de 